\title{
FIRST PASSAGE TIMES OF CONSTANT- ELASTICITY-OF-VARIANCE PROCESSES WITH TWO-SIDED REFLECTING BARRIERS
}

\author{
LIJUN BO ${ }^{* * *}$ AND \\ CHEN HAO, ${ }^{*}$ Xidian University
}

\begin{abstract}
In this paper we explore the first passage times of constant-elasticity-of-variance (CEV) processes with two-sided reflecting barriers. The explicit Laplace transforms of the first passage times are derived. Our results can include analytic formulae concerning Laplace transforms of first passage times of reflected Ornstein-Uhlenbeck processes, reflected geometric Brownian motions, and reflected square-root processes.
\end{abstract}

Keywords: First passage time; CEV process; reflecting barrier

2010 Mathematics Subject Classification: Primary 60J60

Secondary $60 \mathrm{~K} 10$

\section{Introduction}

In this paper we consider the following constant-elasticity-of-variance (CEV) process with reflection at the two side barriers $c$ and $d(0<c<d<+\infty)$ :

$$
\begin{aligned}
& X_{t}=x_{0}+\mu \int_{0}^{t} X_{s} \mathrm{~d} s+\sigma \int_{0}^{t} X_{s}^{\beta+1} \mathrm{~d} W_{s}+L_{t}-U_{t} \in I_{c, d} \text { for all } t>0, \\
& X_{0}=x_{0} \in I_{c, d} .
\end{aligned}
$$

Here the state space $I_{c, d}=[c, d], \mu \neq 0$ is the drift rate, $\sigma>0$ is the volatility scale parameter, and $\beta \leq 0$ is the volatility elasticity parameter. We take as given a filtered probability space $\left(\Omega, \mathcal{F},\left(\mathcal{F}_{t} ; t \geq 0\right), \mathrm{P}\right)$ carrying a standard Brownian motion $W=\left(W_{t} ; t \geq 0\right)$, where the filtration $\left(\mathcal{F}_{t} ; t \geq 0\right)$ satisfies the usual conditions.

The nonnegative processes $L=\left(L_{t} ; t \geq 0\right)$ and $U=\left(U_{t} ; t \geq 0\right)$ are called the regulators of the reflected CEV (RCEV) process at barriers $c$ and $d$, respectively. Moreover, the regulators $L$ and $U$ can be uniquely identified by the following two properties up to a positive constant factor (see [10]).

(A) The nonnegative processes $t \rightarrow L_{t}$ and $t \rightarrow U_{t}$ are nondecreasing and continuous, and $L_{0}=U_{0}=0$.

(B) For all positive $t$, it holds that

$$
L_{t}=\int_{0}^{t} \mathbf{1}_{\left\{X_{s}=c\right\}} \mathrm{d} L_{s} \text { and } U_{t}=\int_{0}^{t} \mathbf{1}_{\left\{X_{s}=d\right\}} \mathrm{d} U_{s} .
$$

As discussed in [6] and [7], the CEV model specifies the instantaneous volatility as a power function $\sigma x^{\beta+1}$ with volatility elasticity parameter $\beta \leq 0$. Hence, the CEV process can cover

Received 29 November 2011; revision received 2 May 2012.

* Postal address: Department of Mathematics, Xidian University, No. 2 South Taibai Road, Xi'an 710071, P. R. China.

** Email address: bolijunnk@gmail.com 
some well-known diffusion processes, such as geometric Brownian motion, the OrnsteinUhlenbeck process, and the square-root process. Correspondingly, our RCEV model (1) can also nest some important reflected diffusion processes. It becomes a reflected geometric Brownian motion (RGBM) when $\beta=0$, a reflected Ornstein-Uhlenbeck (ROU) process when $\beta=-1$, and a reflected square-root process or RCIR process when $\beta=-\frac{1}{2}$. For the volatility elasticity parameter $\beta=-1$, the first passage problems, and the applications in finance and queueing were intensively studied in [2], [3], [4], and [14]; therein the explicit Laplace transforms of the first passage times and relevant integral functionals for ROU processes were obtained. For $\beta=-\frac{1}{2}$, the pathwise uniqueness of the solutions to square-root processes with (skew) reflection at deterministic time-dependent barriers was proved in [13]. In this paper we explore the first passage times of the RCEV process and derive the analytic Laplace transforms corresponding to the first passage times. In addition to the case of the ROU process, our results can include the explicit expressions of the Laplace transforms of the first passage times for the RGBM and RCIR process; to the authors' knowledge, results associated to these two cases do not seem to have been discussed formally in the literature. For the application of the CEV model to option pricing, there does not exist an equivalent martingale measure when $\beta>0$ (see [7] and [11]). However, our formulae concerning the first passage times of the RCEV process can also hold in this case (see Remark 1 of this paper). In addition, the reflected stochastic differential equations can also be applied to model term structures of interest rates and the pricing of barrier options under constraints (see [5] and [9]). These applications associated to the RCEV process will be discussed in future work.

The rest of the paper is organized as follows. We establish a relationship between the regulators and the local times of the RCEV process in Section 2. In Section 3 we present the main Laplace transform results concerning the first passage times of the RCEV process with a formal proof. In the same section we give two corollaries, where the ROU process case and the RCIR process case are respectively discussed. Finally, we plot the images of several abstract Laplace transform functions with respect to the initial value.

\section{Regulators and local times of RCEV processes}

In this section we give a relationship between the local times and the regulators of the RCEV process (1).

First we assume that $X^{0}=\left(X_{t}^{0} ; t \geq 0\right)$ is the CEV process without reflection (i.e. $L=U \equiv$ 0 in (1)). Note that the CEV process $X^{0}=\left(X_{t}^{0} ; t \geq 0\right)$ and the RCEV process $X=\left(X_{t} ; t \geq\right.$ $0)$ are continuous semimartingales. Let $\ell^{a+}(M)=\left(\ell_{t}^{a+}(M) ; t \geq 0\right)$ be the upper local-time process of any continuous semimartingale $M=\left(M_{t} ; t \geq 0\right)$ concentrated at the point $a \in \mathbb{R}$. Then, the upper local-time process $\ell^{a+}(M)$ can be identified by (see [12, Corollary VI.1.9])

$$
\ell_{t}^{a+}(M)=\lim _{\varepsilon \downarrow 0} \frac{1}{\varepsilon} \int_{0}^{t} \mathbf{1}_{\left\{a \leq M_{s}<a+\varepsilon\right\}} \mathrm{d}\langle M, M\rangle_{s}
$$

for all positive $t$. Correspondingly, the lower local time of $M$ at point $a$ is defined as

$$
\ell_{t}^{a-}(M)=\lim _{\varepsilon \downarrow 0} \frac{1}{\varepsilon} \int_{0}^{t} \mathbf{1}_{\left\{a-\varepsilon<M_{s} \leq a\right\}} \mathrm{d}\langle M, M\rangle_{s}, \quad t>0 .
$$

In particular, if $a=0$, the following result shows that the behavior of the upper local time $\ell^{0+}\left(X^{0}\right)$ vanishes when the volatility elasticity parameter $\beta \geq-\frac{1}{2}$.

Lemma 1. Let $\beta \geq-\frac{1}{2}$. Then the upper local-time process $\ell^{0+}\left(X^{0}\right)$ of the CEV process $X^{0}$ vanishes. Moreover, if $\mu<0$ and $X_{0}^{0} \geq 0$, then $X_{t}^{0} \geq 0$, P-almost surely for all positive $t$. 
Proof. When $\beta>-\frac{1}{2}$, the first result in this case follows directly from (2) by setting $a=0$. In fact, for all positive $t$ and $\varepsilon>0$, we have

$$
\frac{1}{\varepsilon} \int_{0}^{t} \mathbf{1}_{\left\{0 \leq X_{s}^{0}<\varepsilon\right\}} \mathrm{d}\left\langle X^{0}, X^{0}\right\rangle_{s}=\frac{\sigma^{2}}{\varepsilon} \int_{0}^{t} \mathbf{1}_{\left\{0 \leq X_{s}^{0}<\varepsilon\right\}} X_{s}^{2 \beta+2} \mathrm{~d} s<\frac{\sigma^{2}}{\varepsilon} \int_{0}^{t} \varepsilon^{2 \beta+2} \mathrm{~d} s=\sigma^{2} \varepsilon^{2 \beta+1} t,
$$

which approaches 0 as $\varepsilon \downarrow 0$ for each fixed $t>0$, since $2 \beta+1>0$. When $\beta=-\frac{1}{2}$ (i.e. the RCIR case), using a similar argument to that used in [13] and the occupation time formula (see, e.g. [12, Corollary VI.1.6]), we have

$$
\begin{aligned}
\int_{\mathbb{R}} \frac{1}{|a|} \mathbf{1}_{\{a \neq 0\}} \ell_{t}^{a+}\left(X^{0}\right) \mathrm{d} a & =\sigma^{2} \int_{0}^{t} \frac{1}{\left|X_{s}^{0}\right|} \mathbf{1}_{\left\{\left|X_{s}^{0}\right|>0\right\}}\left|X_{s}^{0}\right|^{2 \beta+2} \mathrm{~d} s \\
& =\sigma^{2} \int_{0}^{t} \mathbf{1}_{\left\{\left|X_{s}^{0}\right|>0\right\}} \mathrm{d} s \\
& \leq \sigma^{2} t \text { for all } t>0 .
\end{aligned}
$$

Note that $|a|^{-1}$ is not integrable in any neighborhood of $a=0$. Then it must hold that $\ell_{t}^{0+}\left(X^{0}\right)=0$ for all positive $t$.

As for the second assertion, using a similar argument to that used in the proof of Lemma 2.1(ii) of [13], from Tanaka's formula (see, e.g. [12, Theorem VI.1.2]), it follows that

$$
\begin{aligned}
\mathrm{E}\left[\left(X_{t \wedge \tau_{n}}^{0}\right)^{-}\right] & =\mathrm{E}\left[\left(X_{0}^{0}\right)^{-}\right]-\mathrm{E}\left[\int_{0}^{t \wedge \tau_{n}} \mathbf{1}_{\left\{X_{s}^{0} \leq 0\right\}} \mathrm{d} X_{s}^{0}\right]+\frac{1}{2} \mathrm{E}\left[\ell_{t \wedge \tau_{n}}^{0+}\left(X^{0}\right)\right] \\
& =-\mu \mathrm{E}\left[\int_{0}^{t \wedge \tau_{n}} \mathbf{1}_{\left\{X_{s}^{0} \leq 0\right\}} X_{s}^{0} \mathrm{~d} s\right]-\sigma \mathrm{E}\left[\int_{0}^{t \wedge \tau_{n}} \mathbf{1}_{\left\{X_{s}^{0} \leq 0\right\}}\left(X_{s}^{0}\right)^{\beta+1} \mathrm{~d} W_{s}\right] \\
& =-\mu \mathrm{E}\left[\int_{0}^{t \wedge \tau_{n}} \mathbf{1}_{\left\{X_{s}^{0} \leq 0\right\}} X_{s}^{0} \mathrm{~d} s\right] \\
& \leq 0
\end{aligned}
$$

where $\tau_{n}=\inf \left\{t>0 ;\left|X_{t}^{0}\right| \geq n\right\}$ with $n \in \mathbb{N}$. This implies that $X_{t \wedge \tau_{n}}^{0} \geq 0$, P-almost surely for each $n \in \mathbb{N}$. Letting $n \rightarrow \infty$ concludes the proof.

As for the RCEV process $X$, the following proposition presents a relationship between the regulators $L$ and $U$, and the upper local-time process $\ell^{c+}(X)$ and the lower local-time process $\ell^{d-}(X)$, where $0<c<d<+\infty$ are the reflecting barriers of the RECV process $X$.

Proposition 1. The regulators $L$ and $U$ respectively admit, $\mathrm{P}$-almost surely, the equalities

$$
L_{t}=\frac{1}{2} \ell_{t}^{c+}(X) \text { and } U_{t}=\frac{1}{2} \ell_{t}^{d-}(X) \text { for all } t \geq 0,
$$

where $\ell^{c+}(X)$ and $\ell^{d-}(X)$ denote the upper local time and the lower local time of the RCEV process $X$ concentrated at the points $c$ and $d$, respectively.

Proof. We begin by proving the first assertion. Using Tanaka's formula (see, e.g. [12, Theorem VI.1.2]), for the lower reflecting barrier $c>0$, we have

$$
\left(X_{t}-c\right)^{+}=\left(X_{0}-c\right)^{+}+\int_{0}^{t} \mathbf{1}_{\left\{X_{s} \geq c\right\}} \mathrm{d} X_{s}-\int_{0}^{t} \mathbf{1}_{\left\{X_{s}=c\right\}} \mathrm{d} X_{s}+\frac{1}{2} \ell_{t}^{c+}(X) .
$$


Note that $X_{t} \geq c$ for all $t \geq 0$. Then, the above display can be simplified to

$$
X_{t}-c=X_{0}-c+X_{t}-X_{0}-\int_{0}^{t} \mathbf{1}_{\left\{X_{s}=c\right\}} \mathrm{d} X_{s}+\frac{1}{2} \ell_{t}^{c+}(X) .
$$

Hence, for all positive $t$,

$$
\begin{aligned}
\frac{1}{2} \ell_{t}^{c+}(X)= & \mu \int_{0}^{t} \mathbf{1}_{\left\{X_{s}=c\right\}} X_{s} \mathrm{~d} s+\sigma \int_{0}^{t} \mathbf{1}_{\left\{X_{s}=c\right\}} X_{s}^{\beta+1} \mathrm{~d} W_{s}+\int_{0}^{t} \mathbf{1}_{\left\{X_{s}=c\right\}} \mathrm{d} L_{s} \\
& -\int_{0}^{t} \mathbf{1}_{\left\{X_{s}=c\right\}} \mathrm{d} U_{s} \\
= & c \mu \int_{0}^{t} \mathbf{1}_{\left\{X_{s}=c\right\}} \mathrm{d} s+\sigma \int_{0}^{t} \mathbf{1}_{\left\{X_{s}=c\right\}} X_{s}^{\beta+1} \mathrm{~d} W_{s}+L_{t} .
\end{aligned}
$$

For the last equality, we used property (B) concerning the regulators $L$ and $U$. Moreover, we have $\int_{0}^{t} \mathbf{1}_{\left\{X_{s}=c\right\}} X_{s}^{\beta+1} \mathrm{~d} W_{s}=0$ for all positive $t$, since a continuous (local) martingale of finite variation is equal to its initial value. Finally, we obtain the equality

$$
L_{t}=\frac{1}{2} \ell_{t}^{c+}(X)-c \mu \int_{0}^{t} \mathbf{1}_{\left\{X_{s}=c\right\}} \mathrm{d} s \text { for all } t \geq 0 .
$$

We next verify that the time of the RCEV process spent on the lower reflecting barrier $c>0$ has Lebesgue measure 0 . In fact, as a simple consequence of the occupation time formula (see, e.g. [12, Corollary VI.1.6]), we have, P-almost surely,

$$
\int_{0}^{t} \mathbf{1}_{\left\{X_{s}=c\right\}} \mathrm{d}\langle X, X\rangle_{s}=\int_{-\infty}^{\infty} \mathbf{1}_{\{a=c\}} \ell_{t}^{a+}(X) \mathrm{d} a=\ell_{t}^{c+}(X) \int_{-\infty}^{\infty} \mathbf{1}_{\{a=c\}} \mathrm{d} a=0 \quad \text { for all } t \geq 0 .
$$

As a consequence, $\int_{0}^{t} \mathbf{1}_{\left\{X_{s}=c\right\}} \mathrm{d}\langle X, X\rangle_{s}=\sigma c^{2 \beta+2} \int_{0}^{t} \mathbf{1}_{\left\{X_{s}=c\right\}} \mathrm{d} s=0$. Thus, P-almost surely,

$$
\int_{0}^{t} \mathbf{1}_{\left\{X_{s}=c\right\}} \mathrm{d} s=0 \text { for all } t \geq 0
$$

Then the first desired equality in Proposition 1 follows from (4).

As for the second assertion, by Tanaka's formula again, we arrive at

$$
\left(d-X_{t}\right)^{+}=\left(d-X_{0}\right)^{+}-\int_{0}^{t} \mathbf{1}_{\left\{X_{s}<d\right\}} \mathrm{d} X_{s}+\frac{1}{2} \ell_{t}^{(-d)+}(-X),
$$

where $\ell^{(-d)+}(-X)=\left(\ell_{t}^{(-d)+}(-X) ; t \geq 0\right)$ corresponds to the upper local time of the dual process of $X$ at point $-d$. Using similar arguments to those used to derive (4) and (5), we have

$$
U_{t}=\frac{1}{2} \ell_{t}^{(-d)+}(-X) \text { for all } t \geq 0 .
$$

From the definition of the upper local time given in (2), it follows that

$$
\begin{aligned}
\ell_{t}^{(-d)+}(-X) & =\lim _{\varepsilon \downarrow 0} \frac{1}{\varepsilon} \int_{0}^{t} \mathbf{1}_{\left\{-d \leq-X_{s}<-d+\varepsilon\right\}} \mathrm{d}\langle-X,-X\rangle_{s} \\
& =\lim _{\varepsilon \downarrow 0} \frac{1}{\varepsilon} \int_{0}^{t} \mathbf{1}_{\left\{d-\varepsilon<X_{s} \leq d\right\}} \mathrm{d}\langle X, X\rangle_{s} \\
& =\ell_{t}^{d-}(X), \quad t>0,
\end{aligned}
$$

by virtue of the definition of the lower local time given in (3). The validity of the second assertion is thus proved. 


\section{First passage times of RCEV processes}

Our aim in this section is to derive the analytic Laplace transform of the first passage time of the RCEV process $X=\left(X_{t} ; t \geq 0\right)$ given by (1), i.e.

$$
T_{a}=\inf \left\{t>0 ; X_{t}=a\right\}, \quad a \in I_{c, d},
$$

where inf $\varnothing=\infty$ by convention. The corresponding Laplace transform of $T_{a}$ is defined by

$$
\mathbb{L}\left(a, \theta ; x_{0}\right)=\mathrm{E}_{x_{0}}\left[\mathrm{e}^{-\theta T_{a}}\right],
$$

where $a, x_{0} \in I_{c, d}$ and $\theta>0$.

We apply Theorem 2.1 of [4] to compute $\mathbb{L}\left(a, \theta ; x_{0}\right)$ in the RCEV case where $a \in I_{c, d}^{\circ}:=$ $(c, d)$. For the cases in which $a \in \partial I_{c, d}$, we use an approximation scheme to obtain the expressions of $\mathbb{L}\left(a, \theta ; x_{0}\right)$. To this end, we let $\mathcal{A}$ be a second-order differential operator acting on $I_{c, d}^{\circ}$, defined by

$$
\mathcal{A}=\frac{1}{2} \sigma^{2} x^{2 \beta+2} \frac{\mathrm{d}^{2}}{\mathrm{~d} x^{2}}+\mu x \frac{\mathrm{d}}{\mathrm{d} x}, \quad x \in I_{c, d}^{\circ},
$$

where $\beta \leq 0$ and $\mu \neq 0$.

Using a similar argument to that used in the proof of [4, Theorem 2.1], we have the following result concerning $\mathbb{L}\left(a, \theta ; x_{0}\right)$ with $a \in I_{c, d}^{\circ}$ in the RCEV case.

Proposition 2. Let $\theta>0$. Suppose that the functions $f_{\theta}(x)$ and $g_{\theta}(x)$ respectively satisfy the following ordinary differential equations (ODEs) on $I_{c, d}^{\circ}$ :

$$
\begin{gathered}
\mathcal{A} f_{\theta}(x)=\theta f_{\theta}(x) \quad \text { such that } f_{\theta}^{\prime}(c)=0 \\
\text { and } \quad \mathcal{A} g_{\theta}(x)=\theta g_{\theta}(x) \quad \text { such that } g_{\theta}^{\prime}(d)=0 .
\end{gathered}
$$

Here $0<c<d<\infty$ are the reflecting barriers of the RCEV process (1).

Assume that $f_{\theta}(a) \neq 0$ and $g_{\theta}(a) \neq 0$ in the $a \in I_{c, d}^{\circ}$ case. Then, it holds that

$$
\mathbb{L}\left(a, \theta ; x_{0}\right)=\frac{f_{\theta}\left(x_{0}\right)}{f_{\theta}(a)}, \quad c<x_{0} \leq a<d, \quad \mathbb{L}\left(a, \theta ; x_{0}\right)=\frac{g_{\theta}\left(x_{0}\right)}{g_{\theta}(a)}, \quad c<a \leq x_{0}<d .
$$

By virtue of Proposition 2, we have the following main result of this paper.

Theorem 1. (i) Let $\beta<0$. The Laplace transforms of the first passage time $T_{a}$ are given by

$$
\begin{array}{ll}
\mathbb{L}\left(a, \theta ; x_{0}\right)=\frac{\psi_{\theta}\left(x_{0}\right)+c_{1}(\theta) \phi_{\theta}\left(x_{0}\right)}{\psi_{\theta}(a)+c_{1}(\theta) \phi_{\theta}(a)}, & c \leq x_{0} \leq a \leq d, \\
\mathbb{L}\left(a, \theta ; x_{0}\right)=\frac{\psi_{\theta}\left(x_{0}\right)+c_{2}(\theta) \phi_{\theta}\left(x_{0}\right)}{\psi_{\theta}(a)+c_{2}(\theta) \phi_{\theta}(a)}, & c \leq a \leq x_{0} \leq d,
\end{array}
$$

where $\psi_{\theta}(x)$ and $\phi_{\theta}(x)$ are the hypergeometric functions on $I_{c, d}$ defined by

$$
\psi_{\theta}(x)=z(x)^{m-1 / 2} \mathrm{e}^{\epsilon z(x) / 2} \mathbb{M}_{k_{\theta}, m}(z(x)), \quad \phi_{\theta}(x)=z(x)^{m-1 / 2} \mathrm{e}^{\epsilon z(x) / 2} \mathbb{W}_{k_{\theta}, m}(z(x)),
$$

with $\epsilon=\operatorname{sgn}(\mu \beta)$,

$$
z(x)=\frac{|\mu|}{\sigma^{2}|\beta|} x^{-2 \beta} \quad \text { for } x \in I_{c, d}, \quad m=-\frac{1}{4 \beta}, \quad k_{\theta}=\frac{\epsilon}{2}\left(1+\frac{1}{2 \beta}\right)-\frac{\theta}{2|\mu \beta|},
$$


and

$$
\begin{aligned}
c_{1}(\theta)=- & \frac{\psi_{\theta}^{\prime}(c)}{\phi_{\theta}^{\prime}(c)} \\
=- & {\left[\left(\frac{m-1 / 2-k_{\theta}}{z(c)}+\left(\frac{\epsilon}{2}+\frac{1}{2}\right)\right) \mathbb{M}_{k_{\theta}, m}(z(c))+\frac{m+1 / 2+k_{\theta}}{z(c)} \mathbb{M}_{k_{\theta}+1, m}(z(c))\right] } \\
& \times\left[\left(\frac{m-1 / 2-k_{\theta}}{z(c)}+\left(\frac{\epsilon}{2}+\frac{1}{2}\right)\right) \mathbb{W}_{k_{\theta}, m}(z(c))-\frac{1}{z(c)} \mathbb{W}_{k_{\theta}+1, m}(z(c))\right]^{-1}, \\
c_{2}(\theta)=- & \frac{\psi_{\theta}^{\prime}(d)}{\phi_{\theta}^{\prime}(d)} \\
=- & {\left[\left(\frac{m-1 / 2-k_{\theta}}{z(d)}+\left(\frac{\epsilon}{2}+\frac{1}{2}\right)\right) \mathbb{M}_{k_{\theta}, m}(z(d))+\frac{m+1 / 2+k_{\theta}}{z(d)} \mathbb{M}_{k_{\theta}+1, m}(z(d))\right] } \\
& \times\left[\left(\frac{m-1 / 2-k_{\theta}}{z(d)}+\left(\frac{\epsilon}{2}+\frac{1}{2}\right)\right) \mathbb{W}_{k_{\theta}, m}(z(d))-\frac{1}{z(d)} \mathbb{W}_{k_{\theta}+1, m}(z(d))\right]^{-1} .
\end{aligned}
$$

Here $\mathbb{M}_{k_{\theta}, m}(\cdot)$ and $\mathbb{W}_{k_{\theta}, m}(\cdot)$ are Whittaker functions.

(ii) Let $\beta=0$. The Laplace transforms of the first passage time $T_{a}$ are given by

$$
\begin{array}{ll}
\mathbb{L}\left(a, \theta ; x_{0}\right)=\frac{x_{0}^{\theta_{1}}+c_{1}(\theta) x_{0}^{\theta_{2}}}{a^{\theta_{1}}+c_{1}(\theta) a^{\theta_{2}}}, & c \leq x_{0} \leq a \leq d, \\
\mathbb{L}\left(a, \theta ; x_{0}\right)=\frac{x_{0}^{\theta_{1}}+c_{2}(\theta) x_{0}^{\theta_{2}}}{a^{\theta_{1}}+c_{2}(\theta) a^{\theta_{2}}}, \quad c \leq a \leq x_{0} \leq d,
\end{array}
$$

where

$$
\begin{gathered}
c_{1}(\theta)=-\frac{\theta_{1}}{\theta_{2}} c^{\theta_{1}-\theta_{2}}, \quad c_{2}(\theta)=-\frac{\theta_{1}}{\theta_{2}} d^{\theta_{1}-\theta_{2}}, \\
\theta_{1}=\frac{\sigma^{2}-2 \mu+\sqrt{\left(2 \mu-\sigma^{2}\right)^{2}+8 \theta \sigma^{2}}}{2 \sigma^{2}}, \quad \theta_{2}=\frac{\sigma^{2}-2 \mu-\sqrt{\left(2 \mu-\sigma^{2}\right)^{2}+8 \theta \sigma^{2}}}{2 \sigma^{2}} .
\end{gathered}
$$

Proof. We first prove the case in which $a \in I_{c, d}^{\circ}$ by employing Proposition 2. By virtue of Proposition 2, it suffices to find the general solution to the following ODE:

$$
\mathcal{A} f(x)=\theta f(x), \quad x \in I_{c, d}^{\circ} .
$$

In other words, the function $f(x)$ satisfies

$$
\frac{1}{2} \sigma^{2} x^{2 \beta+2} \frac{\mathrm{d}^{2} f}{\mathrm{~d} x^{2}}(x)+\mu x \frac{\mathrm{d} f}{\mathrm{~d} x}(x)=\theta f(x), \quad x \in I_{c, d}^{\circ},
$$

where $\beta \leq 0$ and $\mu \neq 0$.

Case 1: $\beta<0$. We first define $z(x)$ by

$$
z(x)=\frac{|\mu|}{\sigma^{2}|\beta|} x^{-2 \beta}, \quad x \in I_{c, d}^{\circ} .
$$

Substitute the equalities

$$
\frac{\mathrm{d} f}{\mathrm{~d} x}=\frac{\mathrm{d} f}{\mathrm{~d} z} \frac{\mathrm{d} z}{\mathrm{~d} x} \quad \text { and } \quad \frac{\mathrm{d}^{2} f}{\mathrm{~d} x^{2}}=\frac{\mathrm{d}^{2} f}{\mathrm{~d} z^{2}}\left(\frac{\mathrm{d} z}{\mathrm{~d} x}\right)^{2}+\frac{\mathrm{d} f}{\mathrm{~d} z} \frac{\mathrm{d}^{2} z}{\mathrm{~d} x^{2}}
$$


into the ODE (7) to obtain

$$
z \frac{\mathrm{d}^{2} f}{\mathrm{~d} z^{2}}(z)+\left[\left(1+\frac{1}{2 \beta}\right)-\frac{\mu \beta}{|\mu \beta|} z\right] \frac{\mathrm{d} f}{\mathrm{~d} z}(z)-\frac{\theta}{2|\mu \beta|} f(z)=0 .
$$

Define the function $g(z)$ through $f(z)=z^{m-1 / 2} \mathrm{e}^{\epsilon z / 2} g(z)$, with $m=-1 / 4 \beta$ and $\epsilon=\operatorname{sgn}(\mu \beta)$. We find that

$$
\frac{\mathrm{d}^{2} g}{\mathrm{~d} z^{2}}(z)+\left(\frac{1 / 4-m^{2}}{z^{2}}+\frac{k_{\theta}}{z}-\frac{1}{4}\right) g(z)=0,
$$

where

$$
k_{\theta}=\frac{\epsilon}{2}\left(1+\frac{1}{2 \beta}\right)-\frac{\theta}{2|\mu \beta|} .
$$

The ODE (9) is called the Whittaker equation, which has two linearly independent solutions $\mathbb{M}_{k_{\theta}, m}(z)$ and $\mathbb{W}_{k_{\theta}, m}(z)$ (we call $\mathbb{M}(\cdot)$ and $\mathbb{W}(\cdot)$ the Whittaker functions). As a consequence, the general solution to the ODE (7) is given by

$$
\begin{aligned}
f_{\theta}(x) & =\hat{C}_{0} z(x)^{m-1 / 2} \mathrm{e}^{\epsilon z(x) / 2} \mathbb{M}_{k_{\theta}, m}(z(x))+C_{0} z(x)^{m-1 / 2} \mathrm{e}^{\epsilon z(x) / 2} \mathbb{W}_{k_{\theta}, m}(z(x)) \\
& :=\hat{C}_{0} \psi_{\theta}(x)+C_{0} \phi_{\theta}(x),
\end{aligned}
$$

where $\hat{C}_{0}, C_{0} \in \mathbb{R}$ are determined by the boundary conditions. By using the boundary conditions $f_{\theta}^{\prime}(c)=0$ and $g_{\theta}^{\prime}(d)=0$ in Proposition 2 , there exist constants $\hat{C}_{1}, \hat{C}_{2} \in \mathbb{R}$ such that, for $x \in I_{c, d}^{\circ}$,

$$
f_{\theta}(x)=\hat{C}_{1}\left[\psi_{\theta}(x)+c_{1}(\theta) \phi_{\theta}(x)\right] \quad \text { with } \quad c_{1}(\theta)=-\frac{\psi_{\theta}^{\prime}(c)}{\phi_{\theta}^{\prime}(c)}
$$

and

$$
g_{\theta}(x)=\hat{C}_{2}\left[\psi_{\theta}(x)+c_{2}(\theta) \phi_{\theta}(x)\right] \quad \text { with } \quad c_{2}(\theta)=-\frac{\psi_{\theta}^{\prime}(d)}{\phi_{\theta}^{\prime}(d)} .
$$

Then the desired results follow from Proposition 2.

Case 2: $\beta=0$. This case corresponds to the RGBM for the RCEV process (1). This indicates that the ODE (7) is an Eulerian equation. It is known that the general solution to the Eulerian ODE (7) is given by

$$
f_{\theta}(x)=\hat{C}_{0} x^{\theta_{1}}+C_{0} x^{\theta_{2}}, \quad x \in I_{c, d}^{\circ},
$$

where $\theta_{1}$ and $\theta_{2}$ are the eigenvalues of the ODE (7). Accordingly, the desired results follow from Proposition 2.

Finally, we consider the cases in which $a \in \partial I_{c, d}=\{c, d\}$. Here we adopt an approximation scheme to derive the Laplace transforms of the first passage times $T_{c}$ and $T_{d}$ with first hitting levels taken to be reflecting barriers. Take a sequence of increasing positive real numbers $\left(d_{n} ; n \in \mathbb{N}\right)$ such that $\lim _{n \rightarrow \infty} d_{n}=d$, the upper reflecting barrier. Then

$$
T_{d_{n}}=\inf \left\{t>0 ; X_{t} \geq d_{n}\right\}, \quad n \in \mathbb{N},
$$

when the initial value $x_{0} \in[c, d)$. Moreover, it holds that $T_{d_{n}} \uparrow T_{d}$, P-almost surely as $n \rightarrow \infty$. Using the monotone convergence theorem, we arrive at

$$
\mathbb{L}\left(d, \theta ; x_{0}\right)=\mathrm{E}_{x_{0}}\left[\mathrm{e}^{-\theta T_{d}}\right]=\lim _{n \rightarrow \infty} \mathrm{E}_{x_{0}}\left[\mathrm{e}^{-\theta T_{d_{n}}}\right]=\lim _{n \rightarrow \infty} \frac{f_{\theta}\left(x_{0}\right)}{f_{\theta}\left(d_{n}\right)}=\frac{f_{\theta}\left(x_{0}\right)}{f_{\theta}(d)}, \quad x_{0} \in[c, d),
$$


since $f_{\theta}(x)$ is continuous at the point $x=d$. We can similarly deal with the case in which $a=c$; hence, the proof is omitted. This completes the proof of Theorem 1 .

Remark 1. 1. Our results in Theorem 1 also hold when the volatility elasticity parameter $\beta>0$ and $\beta \neq \frac{1}{2}, \frac{1}{4}, \frac{1}{6}, \ldots$ (since the Whittaker function $\mathbb{M}_{k, m}(\cdot)$ does not exist for $2 m=$ $-1,-2,-3, \ldots)$.

2. For the $\beta<0$ case, we can further simplify the results of Theorem 1 according to the sign of the drift parameter $\mu \neq 0$. If $\mu>0$ then the parameter $\epsilon=-1$. Using properties of the derivatives of Whittaker functions, i.e.

$$
\begin{aligned}
& \frac{\mathrm{d}^{n}}{\mathrm{~d} z^{n}}\left[\mathrm{e}^{-z / 2} z^{m-1 / 2} \mathbb{M}_{k, m}(z)\right]=(-1)^{n}(-2 m)_{n} \mathrm{e}^{-z / 2} z^{m-n / 2-1 / 2} \mathbb{M}_{k+n / 2, m-n / 2}(z), \\
& \frac{\mathrm{d}^{n}}{\mathrm{~d} z^{n}}\left[\mathrm{e}^{-z / 2} z^{m-1 / 2} \mathbb{W}_{k, m}(z)\right]=(-1)^{n} \mathrm{e}^{-z / 2} z^{m-n / 2-1 / 2} \mathbb{W}_{k+n / 2, m-n / 2}(z), \quad n \in \mathbb{N},
\end{aligned}
$$

where $(b)_{n}=b(b+1) \cdots(b+n-1)$ and $(b)_{0}=1$, the constants $c_{1}(\theta)$ and $c_{2}(\theta)$ in Theorem 1 can be rewritten as

$$
\begin{aligned}
& c_{1}(\theta)=-\frac{\psi_{\theta}^{\prime}(c)}{\phi_{\theta}^{\prime}(c)}=\frac{2 m \mathbb{M}_{k_{\theta}+1 / 2, m-1 / 2}\left(|\mu| c^{-2 \beta} / \sigma^{2}|\beta|\right)}{\mathbb{W}_{k_{\theta}+1 / 2, m-1 / 2}\left(|\mu| c^{-2 \beta} / \sigma^{2}|\beta|\right)}, \\
& c_{2}(\theta)=-\frac{\psi_{\theta}^{\prime}(d)}{\phi_{\theta}^{\prime}(d)}=\frac{2 m \mathbb{M}_{k_{\theta}+1 / 2, m-1 / 2}\left(|\mu| d^{-2 \beta} / \sigma^{2}|\beta|\right)}{\mathbb{W}_{k_{\theta}+1 / 2, m-1 / 2}\left(|\mu| d^{-2 \beta} / \sigma^{2}|\beta|\right)} .
\end{aligned}
$$

If $\mu<0$ then the parameter $\epsilon=1$. Using the equalities concerning the derivatives for $n \in \mathbb{N}$, i.e.

$$
\begin{aligned}
& \frac{\mathrm{d}^{n}}{\mathrm{~d} z^{n}}\left[\mathrm{e}^{z / 2} z^{m-1 / 2} \mathbb{M}_{k, m}(z)\right]=(-1)^{n}(-2 m)_{n} \mathrm{e}^{z / 2} z^{m-n / 2-1 / 2} \mathbb{M}_{k-n / 2, m-n / 2}(z), \\
& \frac{\mathrm{d}^{n}}{\mathrm{~d} z^{n}}\left[\mathrm{e}^{z / 2} z^{m-1 / 2} \mathbb{W}_{k, m}(z)\right]=(-1)^{n}\left(\frac{1}{2}-m-k\right)_{n} \mathrm{e}^{z / 2} z^{m-n / 2-1 / 2} \mathbb{W}_{k-n / 2, m-n / 2}(z),
\end{aligned}
$$

the constants $c_{1}(\theta)$ and $c_{2}(\theta)$ can be rewritten as

$$
\begin{aligned}
& c_{1}(\theta)=-\frac{\psi_{\theta}^{\prime}(c)}{\phi_{\theta}^{\prime}(c)}=\frac{2 m \mathbb{M}_{k_{\theta}-1 / 2, m-1 / 2}\left(|\mu| c^{-2 \beta} / \sigma^{2}|\beta|\right)}{\left(1 / 2-m-k_{\theta}\right) \mathbb{W}_{k_{\theta}-1 / 2, m-1 / 2}\left(|\mu| c^{-2 \beta} / \sigma^{2}|\beta|\right)}, \\
& c_{2}(\theta)=-\frac{\psi_{\theta}^{\prime}(d)}{\phi_{\theta}^{\prime}(d)}=\frac{2 m \mathbb{M}_{k_{\theta}-1 / 2, m-1 / 2}\left(|\mu| d^{-2 \beta} / \sigma^{2}|\beta|\right)}{\left(1 / 2-m-k_{\theta}\right) \mathbb{W}_{k_{\theta}-1 / 2, m-1 / 2}\left(|\mu| d^{-2 \beta} / \sigma^{2}|\beta|\right)} .
\end{aligned}
$$

3. For the case in which $\beta<0, \mu<0$, and $\eta:=1+1 / 2 \beta \neq 0,-1,-2, \ldots$, the ODE (8) corresponds to a Kummer equation. Hence, the Laplace transforms of the first passage time $T_{a}$ can be expressed as

$$
\begin{array}{ll}
\mathbb{L}\left(a, \theta ; x_{0}\right)=\frac{\mathbb{M}\left(a_{\theta}, \eta, z\left(x_{0}\right)\right)+c_{1}(\theta) \mathbb{U}\left(a_{\theta}, \eta, z\left(x_{0}\right)\right)}{\mathbb{M}\left(a_{\theta}, \eta, z(a)\right)+c_{1}(\theta) \mathbb{U}\left(a_{\theta}, \eta, z(a)\right)}, & c \leq x_{0} \leq a \leq d, \\
\mathbb{L}\left(a, \theta ; x_{0}\right)=\frac{\mathbb{M}\left(a_{\theta}, \eta, z\left(x_{0}\right)\right)+c_{2}(\theta) \mathbb{U}\left(a_{\theta}, \eta, z\left(x_{0}\right)\right)}{\mathbb{M}\left(a_{\theta}, \eta, z(a)\right)+c_{2}(\theta) \mathbb{U}\left(a_{\theta}, \eta, z(a)\right)}, & c \leq a \leq x_{0} \leq d,
\end{array}
$$

where

$$
c_{1}(\theta)=-\frac{\mathbb{M}^{\prime}\left(a_{\theta}, \eta,|\mu| c^{-2 \beta} / \sigma^{2}|\beta|\right)}{\mathbb{U}^{\prime}\left(a_{\theta}, \eta,|\mu| c^{-2 \beta} / \sigma^{2}|\beta|\right)} \quad \text { and } \quad c_{2}(\theta)=-\frac{\mathbb{M}^{\prime}\left(a_{\theta}, \eta,|\mu| d^{-2 \beta} / \sigma^{2}|\beta|\right)}{\mathbb{U}^{\prime}\left(a_{\theta}, \eta,|\mu| d^{-2 \beta} / \sigma^{2}|\beta|\right)} .
$$


Here $\mathbb{M}(\cdot, \cdot, \cdot)$ and $\mathbb{U}(\cdot, \cdot, \cdot)$ are Kummer functions, $\mathbb{M}^{\prime}(\cdot)$ and $\mathbb{U}^{\prime}(\cdot)$ correspond to partial derivatives with respect to the third variable of the Kummer functions $\mathbb{M}(\cdot, \cdot, \cdot)$ and $\mathbb{U}(\cdot, \cdot, \cdot)$, and the parameters

$$
a_{\theta}=\frac{\theta}{2|\mu \beta|} \quad \text { and } \quad z(x)=\frac{|\mu|}{\sigma^{2}|\beta|} x^{-2 \beta} \quad \text { for } x \in I_{c, d} .
$$

Moreover, by virtue of the differential properties of Kummer functions, i.e.

$$
\begin{aligned}
\frac{\mathrm{d}^{n}}{\mathrm{~d} z^{n}} \mathbb{M}(a, \eta, z) & =\frac{(a)_{n}}{(\eta)_{n}} \mathbb{M}(a+n, \eta+n, z), \\
\frac{\mathrm{d}^{n}}{\mathrm{~d} z^{n}} \mathbb{U}(a, \eta, z) & =(-1)^{n}(a)_{n} \mathbb{U}(a+n, \eta+n, z),
\end{aligned}
$$

it follows that

$$
\begin{aligned}
& c_{1}(\theta)=\frac{\mathbb{M}\left(a_{\theta}+1, \eta+1,|\mu| c^{-2 \beta} / \sigma^{2}|\beta|\right)}{\eta \mathbb{U}\left(a_{\theta}+1, \eta+1,|\mu| c^{-2 \beta} / \sigma^{2}|\beta|\right)}, \\
& c_{2}(\theta)=\frac{\mathbb{M}\left(a_{\theta}+1, \eta+1,|\mu| d^{-2 \beta} / \sigma^{2}|\beta|\right)}{\eta \mathbb{U}\left(a_{\theta}+1, \eta+1,|\mu| d^{-2 \beta} / \sigma^{2}|\beta|\right)} .
\end{aligned}
$$

Based on Theorem 1 and Remark 1, we have the following corollaries.

Corollary 1. (ROU case.) Let $\beta=-1$. Then the Laplace transforms of the first passage time $T_{a}$ are given by

$$
\mathbb{L}\left(a, \theta ; x_{0}\right)=\frac{z\left(x_{0}\right)^{-1 / 4} \mathrm{e}^{\epsilon z\left(x_{0}\right) / 2} \mathbb{M}_{k_{\theta}, 1 / 4}\left(z\left(x_{0}\right)\right)+c_{1}(\theta) z\left(x_{0}\right)^{-1 / 4} \mathrm{e}^{\epsilon z\left(x_{0}\right) / 2} \mathbb{W}_{k_{\theta}, 1 / 4}\left(z\left(x_{0}\right)\right)}{z(a)^{-1 / 4} \mathrm{e}^{\epsilon z(a) / 2} \mathbb{M}_{k_{\theta}, 1 / 4}(z(a))+c_{1}(\theta) z(a)^{-1 / 4} \mathrm{e}^{\epsilon z(a) / 2} \mathbb{W}_{k_{\theta}, 1 / 4}(z(a))},
$$

where $c \leq x_{0} \leq a \leq d$, and

$$
\mathbb{L}\left(a, \theta ; x_{0}\right)=\frac{z\left(x_{0}\right)^{-1 / 4} \mathrm{e}^{\epsilon z\left(x_{0}\right) / 2} \mathbb{M}_{k_{\theta}, 1 / 4}\left(z\left(x_{0}\right)\right)+c_{2}(\theta) z\left(x_{0}\right)^{-1 / 4} \mathrm{e}^{\epsilon z\left(x_{0}\right) / 2} \mathbb{W}_{k_{\theta}, 1 / 4}\left(z\left(x_{0}\right)\right)}{z(a)^{-1 / 4} \mathrm{e}^{\epsilon z(a) / 2} \mathbb{M}_{k_{\theta}, 1 / 4}(z(a))+c_{2}(\theta) z(a)^{-1 / 4} \mathrm{e}^{\epsilon z(a) / 2} \mathbb{W}_{k_{\theta}, 1 / 4}(z(a))},
$$

where $c \leq a \leq x_{0} \leq d$. Here the constants

$$
\begin{aligned}
c_{1}(\theta)=-[ & \left(\frac{1}{z(c)}\left(\frac{\theta}{2|\mu|}-\frac{\epsilon+1}{4}\right)+\frac{\epsilon+1}{2}\right) \mathbb{M}_{k_{\theta}, 1 / 4}(z(c)) \\
& \left.+\left(\frac{\epsilon+3}{4}-\frac{\theta}{2|\mu|}\right) \frac{\mathbb{M}_{k_{\theta}+1,1 / 4}(z(c))}{z(c)}\right] \\
& \times\left[\left(\frac{1}{z(c)}\left(\frac{\theta}{2|\mu|}-\frac{\epsilon+1}{4}\right)+\frac{\epsilon+1}{2}\right) \mathbb{W}_{k_{\theta}, 1 / 4}(z(c))-\frac{\mathbb{W}_{k_{\theta}+1,1 / 4}(z(c))}{z(c)}\right]^{-1}, \\
c_{2}(\theta)=- & \left(\frac{1}{z(d)}\left(\frac{\theta}{2|\mu|}-\frac{\epsilon+1}{4}\right)+\frac{\epsilon+1}{2}\right) \mathbb{M}_{k_{\theta}, 1 / 4}(z(d)) \\
& \left.+\left(\frac{\epsilon+3}{4}-\frac{\theta}{2|\mu|}\right) \frac{\mathbb{M}_{k_{\theta}+1,1 / 4}(z(d))}{z(d)}\right] \\
\times & {\left[\left(\frac{1}{z(d)}\left(\frac{\theta}{2|\mu|}-\frac{\epsilon+1}{4}\right)+\frac{\epsilon+1}{2}\right) \mathbb{W}_{k_{\theta}, 1 / 4}(z(d))-\frac{\mathbb{W}_{k_{\theta}+1,1 / 4}(z(d))}{z(d)}\right]^{-1}, }
\end{aligned}
$$


with

$$
\epsilon=-\operatorname{sgn}(\mu), \quad k_{\theta}=\frac{\epsilon}{4}-\frac{\theta}{2|\mu|}, \quad \text { and } \quad z(x)=\frac{|\mu|}{\sigma^{2}} x^{2} \quad \text { for } x \in I_{c, d} .
$$

Moreover, if $\mu>0$ then the corresponding Laplace transforms are given by taking $\epsilon=-1$ in (10) and (11), respectively. The corresponding constants are given by

$$
c_{1}(\theta)=\frac{\mathbb{M}_{1 / 4-\theta / 2 \mu,-1 / 4}\left(\mu c^{2} / \sigma^{2}\right)}{2 \mathbb{W}_{1 / 4-\theta / 2 \mu,-1 / 4}\left(\mu c^{2} / \sigma^{2}\right)} \quad \text { and } \quad c_{2}(\theta)=\frac{\mathbb{M}_{1 / 4-\theta / 2 \mu,-1 / 4}\left(\mu d^{2} / \sigma^{2}\right)}{2 \mathbb{W}_{1 / 4-\theta / 2 \mu,-1 / 4}\left(\mu d^{2} / \sigma^{2}\right)}
$$

If $\mu<0$, the corresponding Laplace transform can be obtained by taking $\epsilon=1$ in (10) and (11), respectively. The corresponding constants $c_{1}(\theta)$ and $c_{2}(\theta)$ in this case are given by

$$
\begin{gathered}
c_{1}(\theta)=\frac{|\mu| \mathbb{M}_{-\theta / 2|\mu|-1 / 4,-1 / 4}\left(|\mu| c^{2} / \sigma^{2}\right)}{\theta \mathbb{W}_{-\theta / 2|\mu|-1 / 4,-1 / 4}\left(|\mu| c^{2} / \sigma^{2}\right)} \\
\text { and } \quad c_{2}(\theta)=\frac{|\mu| \mathbb{M}_{-\theta / 2|\mu|-1 / 4,-1 / 4}\left(|\mu| d^{2} / \sigma^{2}\right)}{\theta \mathbb{W}_{-\theta / 2|\mu|-1 / 4,-1 / 4}\left(|\mu| d^{2} / \sigma^{2}\right)} .
\end{gathered}
$$

In particular, for the case in which $\mu<0$, we can also express the Laplace transforms by virtue of Kummer functions, i.e.

$$
\mathbb{L}\left(a, \theta ; x_{0}\right)=\frac{\mathbb{M}\left(\theta / 2|\mu|, 1 / 2,|\mu| x_{0}^{2} / \sigma^{2}\right)+c_{1}(\theta) \mathbb{U}\left(\theta / 2|\mu|, 1 / 2,|\mu| x_{0}^{2} / \sigma^{2}\right)}{\mathbb{M}\left(\theta / 2|\mu|, 1 / 2,|\mu| a^{2} / \sigma^{2}\right)+c_{1}(\theta) \mathbb{U}\left(\theta / 2|\mu|, 1 / 2,|\mu| a^{2} / \sigma^{2}\right)}
$$

for $c \leq x_{0} \leq a \leq d$ and

$$
\mathbb{L}\left(a, \theta ; x_{0}\right)=\frac{\mathbb{M}\left(\theta / 2|\mu|, 1 / 2,|\mu| x_{0}^{2} / \sigma^{2}\right)+c_{2}(\theta) \mathbb{U}\left(\theta / 2|\mu|, 1 / 2,|\mu| x_{0}^{2} / \sigma^{2}\right)}{\mathbb{M}\left(\theta / 2|\mu|, 1 / 2,|\mu| a^{2} / \sigma^{2}\right)+c_{2}(\theta) \mathbb{U}\left(\theta / 2|\mu|, 1 / 2,|\mu| a^{2} / \sigma^{2}\right)}
$$

for $c \leq a \leq x_{0} \leq d$, where

$$
c_{1}(\theta)=\frac{2 \mathbb{M}\left(\theta / 2|\mu|+1,3 / 2,|\mu| c^{2} / \sigma^{2}\right)}{\mathbb{U}\left(\theta / 2|\mu|+1,3 / 2,|\mu| c^{2} / \sigma^{2}\right)} \quad \text { and } \quad c_{2}(\theta)=\frac{2 \mathbb{M}\left(\theta / 2|\mu|+1,3 / 2,|\mu| d^{2} / \sigma^{2}\right)}{\mathbb{U}\left(\theta / 2|\mu|+1,3 / 2,|\mu| d^{2} / \sigma^{2}\right)} .
$$

Remark 2. For the ROU case, the lower reflecting barrier can be taken to be $c=0$, as in [2], [3], [4], and [14]. In this case, the constant $c_{1}(\theta)$ should be treated as the limit of the above $c_{1}(\theta)$ when $c \downarrow 0$. Noting that, as $z \rightarrow 0$,

$$
\begin{gathered}
\mathbb{M}(a, b, z)=1+O(z), \quad b \neq 0,-1,-2, \ldots, \\
\mathbb{U}(a, b, z)=\frac{\Gamma(b-1)}{\Gamma(a)} z^{1-b}+\frac{\Gamma(1-b)}{\Gamma(a-b+1)}+O\left(z^{2-b}\right), \quad 1<b<2,
\end{gathered}
$$

then it holds that

$$
c_{1}(\theta)=\frac{2 \mathbb{M}\left(\theta / 2|\mu|+1,3 / 2,|\mu| c^{2} / \sigma^{2}\right)}{\mathbb{U}\left(\theta / 2|\mu|+1,3 / 2,|\mu| c^{2} / \sigma^{2}\right)} \rightarrow 0 \quad \text { as } c \rightarrow 0 .
$$

This implies that if the lower reflecting barrier $c=0$ then the Laplace transform

$$
\mathbb{L}\left(a, \theta ; x_{0}\right)=\frac{\mathbb{M}\left(\theta / 2|\mu|, 1 / 2,|\mu| x_{0}^{2} / \sigma^{2}\right)}{\mathbb{M}\left(\theta / 2|\mu|, 1 / 2,|\mu| a^{2} / \sigma^{2}\right)}, \quad 0 \leq x_{0} \leq a \leq d,
$$


which covers the corresponding formula, Equation (2.15), in [3]. In fact, the function

$$
f_{\theta}\left(x_{0}\right)=\mathbb{M}\left(\frac{\theta}{2|\mu|}, \frac{1}{2}, \frac{|\mu|}{\sigma^{2}} x_{0}^{2}\right)
$$

is the solution to the $\operatorname{ODE}(7)$ with the boundary condition $f_{\theta}^{\prime}(0)=0$.

Corollary 2. (RCIR case.) Let $\beta=-\frac{1}{2}$. Then the Laplace transforms of the first passage time $T_{a}$ are given by

$$
\mathbb{L}\left(a, \theta ; x_{0}\right)=\frac{\mathrm{e}^{\epsilon|\mu| x_{0} / \sigma^{2}} \mathbb{M}_{-\theta /|\mu|, 1 / 2}\left(2|\mu| x_{0} / \sigma^{2}\right)+c_{1}(\theta) \mathrm{e}^{\epsilon|\mu| x_{0} / \sigma^{2}} \mathbb{W}_{-\theta /|\mu|, 1 / 2}\left(2|\mu| x_{0} / \sigma^{2}\right)}{\mathrm{e}^{\epsilon|\mu| a / \sigma^{2}} \mathbb{M}_{-\theta /|\mu|, 1 / 2}\left(2|\mu| a / \sigma^{2}\right)+c_{1}(\theta) \mathrm{e}^{\epsilon|\mu| a / \sigma^{2}} \mathbb{W}_{-\theta /|\mu|, 1 / 2}\left(2|\mu| a / \sigma^{2}\right)},
$$

where $c \leq x_{0} \leq a \leq d$, and

$$
\mathbb{L}\left(a, \theta ; x_{0}\right)=\frac{\mathrm{e}^{\epsilon|\mu| x_{0} / \sigma^{2}} \mathbb{M}_{-\theta /|\mu|, 1 / 2}\left(2|\mu| x_{0} / \sigma^{2}\right)+c_{2}(\theta) \mathrm{e}^{\epsilon|\mu| x_{0} / \sigma^{2}} \mathbb{W}_{-\theta /|\mu|, 1 / 2}\left(2|\mu| x_{0} / \sigma^{2}\right)}{\mathrm{e}^{\epsilon|\mu| a / \sigma^{2}} \mathbb{M}_{-\theta /|\mu|, 1 / 2}\left(2|\mu| a / \sigma^{2}\right)+c_{2}(\theta) \mathrm{e}^{\epsilon|\mu| a / \sigma^{2}} \mathbb{W}_{-\theta /|\mu|, 1 / 2}\left(2|\mu| a / \sigma^{2}\right)}
$$

where $c \leq a \leq x_{0} \leq d$. Here

$$
\begin{aligned}
c_{1}(\theta)=- & {\left[\left(\frac{\epsilon+1}{2}+\frac{\sigma^{2} \theta}{2 \mu^{2} c}\right) \mathbb{M}_{-\theta /|\mu|, 1 / 2}\left(\frac{2|\mu|}{\sigma^{2}} c\right)\right.} \\
& \left.+\left(1-\frac{\theta}{|\mu|}\right) \frac{\sigma^{2}}{2|\mu| c} \mathbb{M}_{1-\theta /|\mu|, 1 / 2}\left(\frac{2|\mu|}{\sigma^{2}} c\right)\right] \\
& \times\left[\left(\frac{\epsilon+1}{2}+\frac{\sigma^{2} \theta}{2 \mu^{2} c}\right) \mathbb{W}_{-\theta /|\mu|, 1 / 2}\left(\frac{2|\mu|}{\sigma^{2}} c\right)-\frac{\sigma^{2}}{2|\mu| c} \mathbb{W}_{1-\theta /|\mu|, 1 / 2}\left(\frac{2|\mu|}{\sigma^{2}} c\right)\right]^{-1}, \\
c_{2}(\theta)=- & {\left[\left(\frac{\epsilon+1}{2}+\frac{\sigma^{2} \theta}{2 \mu^{2} d}\right) \mathbb{M}_{-\theta /|\mu|, 1 / 2}\left(\frac{2|\mu|}{\sigma^{2}} d\right)\right.} \\
& \left.+\left(1-\frac{\theta}{|\mu|}\right) \frac{\sigma^{2}}{2|\mu| d} \mathbb{M}_{1-\theta /|\mu|, 1 / 2}\left(\frac{2|\mu|}{\sigma^{2}} d\right)\right] \\
& \times\left[\left(\frac{\epsilon+1}{2}+\frac{\sigma^{2} \theta}{2 \mu^{2} d}\right) \mathbb{W}_{-\theta /|\mu|, 1 / 2}\left(\frac{2|\mu|}{\sigma^{2}} d\right)-\frac{\sigma^{2}}{2|\mu| d} \mathbb{W}_{1-\theta /|\mu|, 1 / 2}\left(\frac{2|\mu|}{\sigma^{2}} d\right)\right]^{-1},
\end{aligned}
$$

with the parameter $\epsilon=-\operatorname{sgn}(\mu)$.

Moreover if $\mu>0$ then the Laplace transform $\mathbb{L}\left(a, \theta ; x_{0}\right)$ can be obtained by taking $\epsilon=-1$ in (12) and (13), respectively. The corresponding constants are given by

$$
c_{1}(\theta)=\frac{\mathbb{M}_{1 / 2-\theta / \mu, 0}\left(2 \mu c / \sigma^{2}\right)}{\mathbb{W}_{1 / 2-\theta / \mu, 0}\left(2 \mu c / \sigma^{2}\right)} \quad \text { and } \quad c_{2}(\theta)=\frac{\mathbb{M}_{1 / 2-\theta / \mu, 0}\left(2 \mu d / \sigma^{2}\right)}{\mathbb{W}_{1 / 2-\theta / \mu, 0}\left(2 \mu d / \sigma^{2}\right)} .
$$

If $\mu<0$ then the Laplace transform $\mathbb{L}\left(a, \theta ; x_{0}\right)$ can be obtained by taking $\epsilon=1$ in (12) and (13), respectively. The corresponding constants are given by

$$
c_{1}(\theta)=\frac{|\mu| \mathbb{M}_{-\theta /|\mu|-1 / 2,0}\left(2|\mu| c / \sigma^{2}\right)}{\theta \mathbb{W}_{-\theta /|\mu|-1 / 2,0}\left(2|\mu| c / \sigma^{2}\right)} \quad \text { and } \quad c_{2}(\theta)=\frac{|\mu| \mathbb{M}_{-\theta /|\mu|-1 / 2,0}\left(2|\mu| d / \sigma^{2}\right)}{\theta \mathbb{W}_{-\theta /|\mu|-1 / 2,0}\left(2|\mu| d / \sigma^{2}\right)}
$$


We make the following remark concerning the lower reflecting barrier $c=0$. (Note that the regulator $L$ in (1) vanishes when the CEV $\beta \geq-\frac{1}{2}$ by Lemma 1 and Proposition 1.)

Remark 3. Let $\mu<0$. Recall the definition of $c_{1}(\theta)$ given in part 3 of Remark 1. When $\beta<-\frac{1}{2}$ (i.e. $1<\eta+1=2+1 / 2 \beta<2$ ), we find that $c_{1}(\theta) \rightarrow 0$ if the lower reflecting barrier $c$ approaches 0 . Hence, for the case in which $c=0$,

$$
\mathbb{L}\left(a, \theta ; x_{0}\right)=\frac{\mathbb{M}\left(\theta / 2|\mu \beta|, 1+1 / 2 \beta,|\mu| x_{0}^{-2 \beta} / \sigma^{2}|\beta|\right)}{\mathbb{M}\left(\theta / 2|\mu \beta|, 1+1 / 2 \beta,|\mu| a^{-2 \beta} / \sigma^{2}|\beta|\right)}, \quad 0 \leq x_{0} \leq a \leq d .
$$

Recall the definition of $c_{1}(\theta)$ given in part 2 of Remark 1 . When $-\frac{1}{2} \leq \beta<0$, we have $c_{1}(\theta) \rightarrow 0$ as the lower reflecting barrier $c \rightarrow 0$. So in the case in which $c=0$,

$$
\mathbb{L}\left(a, \theta ; x_{0}\right)=\frac{z\left(x_{0}\right)^{m-1 / 2} \mathrm{e}^{z\left(x_{0}\right) / 2} \mathbb{M}_{k_{\theta}, m}\left(z\left(x_{0}\right)\right)}{z(a)^{m-1 / 2} \mathrm{e}^{z(a) / 2} \mathbb{M}_{k_{\theta}, m}(z(a))}, \quad 0 \leq x_{0} \leq a \leq d .
$$

We note that, when $-\frac{1}{2} \leq \beta<0$, the point $\{0\}$ is an absorbing state for $X$ by employing [1, Proposition 2.4]. This implies that $X_{t}=0$ for all positive $t$ if the initial value $x_{0}=0$ in this case. Hence, the Laplace transform of the first passage time $T_{a}$ with $a>0$ is $\mathbb{L}(a, \theta ; 0)=0$, since $T_{a}=+\infty$, which can be covered by (15). When $\beta<-\frac{1}{2}$, the point $\{0\}$ is an instantaneous reflecting state for $X$ by [1, Proposition 2.4] again. Using (14), we have

$$
\mathbb{L}(a, \theta ; 0)=\frac{1}{\mathbb{M}\left(\theta / 2|\mu \beta|, 1+1 / 2 \beta,|\mu| a^{-2 \beta} / \sigma^{2}|\beta|\right)}, \quad 0<a \leq d ;
$$

see also [8].

Let $a \in \partial I_{c, d}$, i.e. the first hitting level is taken as the reflecting barriers. From the definition of the Laplace transform $\mathbb{L}\left(a, \theta ; x_{0}\right)$ given in $(6)$, the Laplace transform function $x_{0} \rightarrow \mathbb{L}\left(d, \theta ; x_{0}\right)$ is increasing on the state space $I_{c, d}$. The Laplace transform function $x_{0} \rightarrow \mathbb{L}\left(c, \theta ; x_{0}\right)$ is decreasing on $I_{c, d}$. We now plot the images of the Laplace transform function $x_{0} \rightarrow \mathbb{L}\left(a, \theta ; x_{0}\right)$ with the first hitting level $a \in \partial I_{c, d}$ in the RGBM, ROU, and RCIR cases by respectively employing Theorem 1 and Corollaries 1 and 2 , and verify the monotonicity of their Laplace transform functions $x_{0} \rightarrow \mathbb{L}\left(a, \theta ; x_{0}\right)$ on $I_{c, d}$.

Here we take the lower reflecting barrier $c=0.5$ and the upper reflecting barrier $d=1$. In addition, the drift rate and the volatility scale parameter are taken to be $\mu=-1$ and $\sigma=1$. We plot the images of the Laplace transform functions $\mathbb{L}\left(c, 1 ; x_{0}\right)$ and $\mathbb{L}\left(d, 1 ; x_{0}\right)$ evaluated at $\theta=1$ with respect to the initial value $x_{0} \in I_{c, d}$. In Figures 1,2 , and 3 we respectively display the monotonicity of the Laplace transform functions $x_{0} \rightarrow \mathbb{L}\left(1,1 ; x_{0}\right)$ and $x_{0} \rightarrow \mathbb{L}\left(0.5,1 ; x_{0}\right)$ in the RGBM, ROU, and RCIR cases. From these figures, we observe that the corresponding displays of $x_{0} \rightarrow \mathbb{L}\left(1,1 ; x_{0}\right)$ are all increasing on $I_{c, d}=[0.5,1]$ and those of $x_{0} \rightarrow \mathbb{L}\left(0.5,1 ; x_{0}\right)$ are all decreasing on $I_{c, d}$. These observations are consistent with the above discussion concerning the monotonicity of the Laplace transform function $x_{0} \rightarrow \mathbb{L}\left(a, \theta ; x_{0}\right)$ with $a \in \partial I_{c, d}$.

Let $a \in I_{c, d}^{\circ}$, and let $\theta>0$ be fixed. By the definition of the Laplace transform $\mathbb{L}\left(a, \theta ; x_{0}\right)$ given in (6), we can see that if the initial value $x_{0}<a$ then $x_{0} \rightarrow \mathbb{L}\left(a, \theta ; x_{0}\right)$ is increasing on $[c, a]$ and is decreasing on $[a, d]$ if $x_{0} \geq a$. We take the same set of parameters as in the $a \in \partial I_{c, d}$ case above, i.e. $(c, d, \mu, \sigma)=(0.5,1,-1,1)$, and then plot the images of the Laplace transform function $x_{0} \rightarrow \mathbb{L}\left(0.75,1 ; x_{0}\right)$ with hitting level $a=0.75$ on the domains $x_{0} \in[0.5,0.75]$ and $x_{0} \in[0.75,1]$ in the RGBM, ROU, and RCIR cases. In Figures 4,5 , and 6 we respectively display the Laplace transform functions on the state space $I_{c, d}=[0.5,1]$. We observe that 

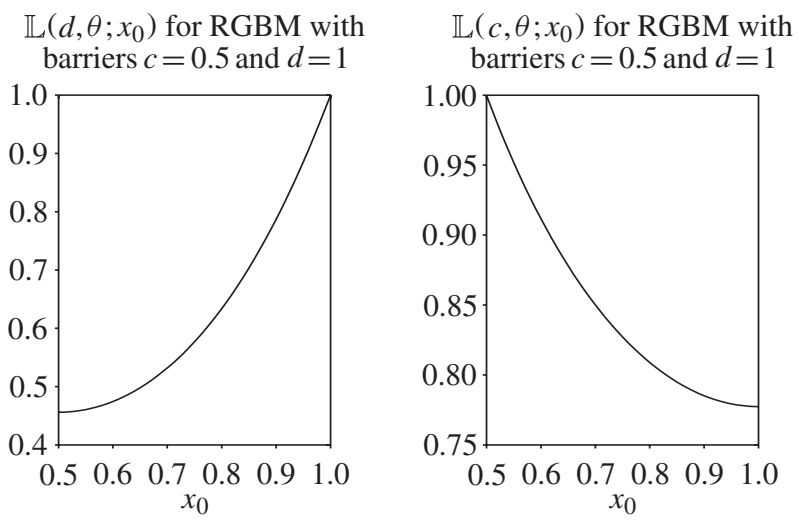

FIGURE 1: The Laplace transform functions $x_{0} \rightarrow \mathbb{L}\left(1,1 ; x_{0}\right)$ (left $)$ and $x_{0} \rightarrow \mathbb{L}\left(0.5,1 ; x_{0}\right)$ (right).
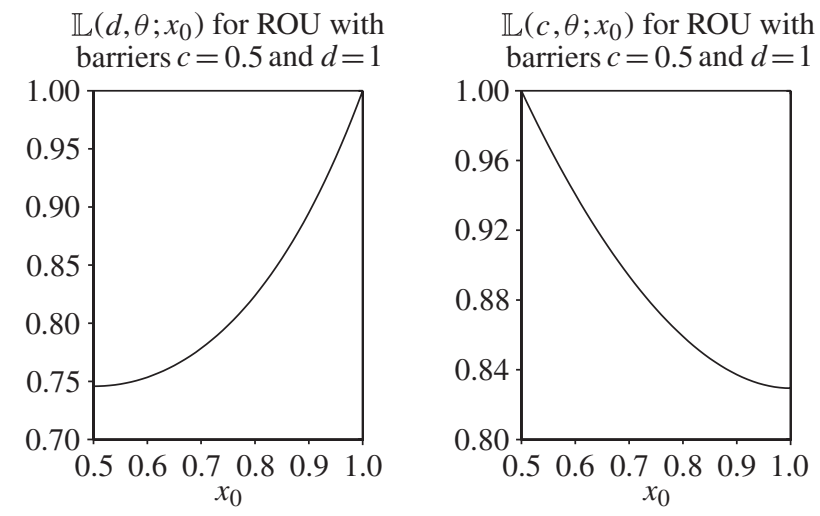

FIGURE 2: The Laplace transform functions $x_{0} \rightarrow \mathbb{L}\left(1,1 ; x_{0}\right)($ left $)$ and $x_{0} \rightarrow \mathbb{L}\left(0.5,1 ; x_{0}\right)$ (right).

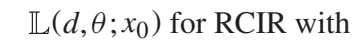
barriers $c=0.5$ and $d=1$

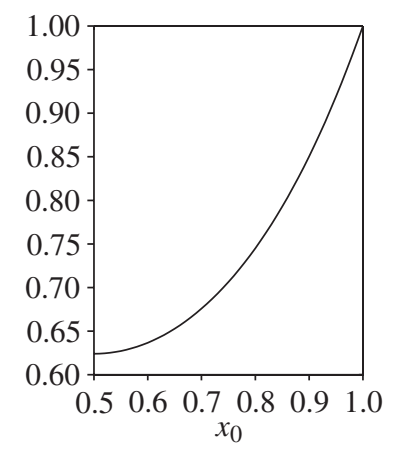

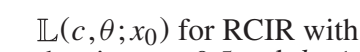

$$
\text { barriers } c=0.5 \text { and } d=1
$$

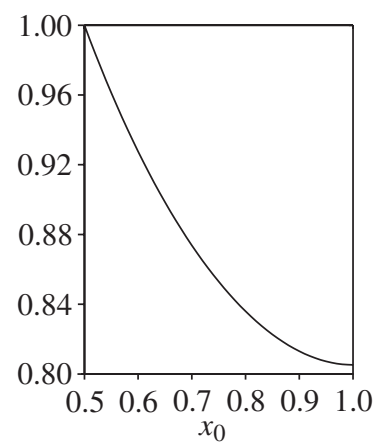

FIGURE 3: The Laplace transform functions $x_{0} \rightarrow \mathbb{L}\left(1,1 ; x_{0}\right)$ (left $)$ and $x_{0} \rightarrow \mathbb{L}\left(0.5,1 ; x_{0}\right)$ (right). 
$\mathbb{L}\left(0.75, \theta ; x_{0}\right)$ for RGBM with barriers $c=0.5$ and $d=1$

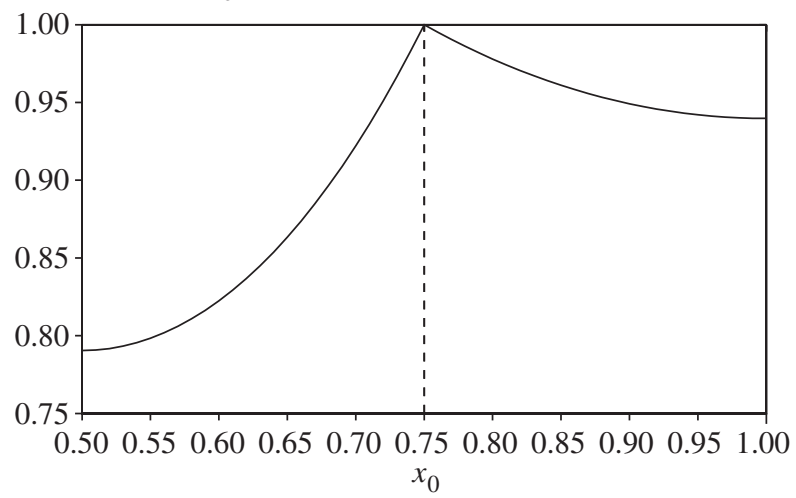

FIGURE 4: The Laplace transform function $x_{0} \rightarrow \mathbb{L}\left(0.75,1 ; x_{0}\right)$ on the domains $[0.5,0.75]$ and $[0.75,1]$.

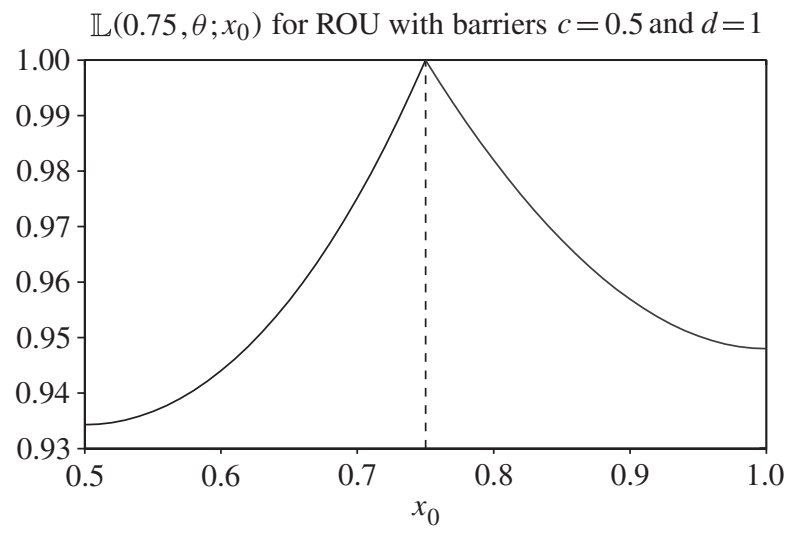

Figure 5: The Laplace transform function $x_{0} \rightarrow \mathbb{L}\left(0.75,1 ; x_{0}\right)$ on the domains $[0.5,0.75]$ and $[0.75,1]$.

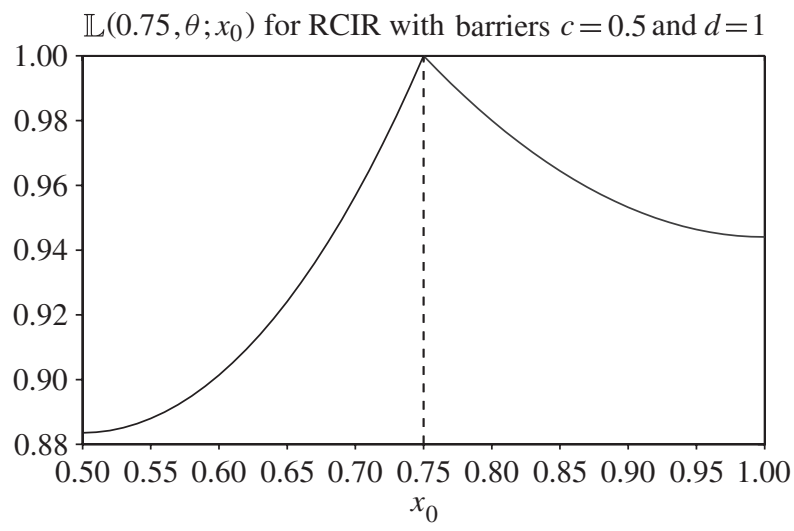

Figure 6: The Laplace transform function $x_{0} \rightarrow \mathbb{L}\left(0.75,1 ; x_{0}\right)$ on the domains $[0.5,0.75]$ and $[0.75,1]$. 
the corresponding displays of $x_{0} \rightarrow \mathbb{L}\left(0.75,1 ; x_{0}\right)$ are all increasing on $[0.5,0.75]$ and are all decreasing on $[0.75,1]$ in the RGBM, ROU, and RCIR cases. These observations are also consistent with the above discussion concerning the monotonicity of $x_{0} \rightarrow \mathbb{L}\left(a, \theta ; x_{0}\right)$ with the first hitting level $a \in I_{c, d}^{\circ}$.

\section{Acknowledgements}

The authors would like to thank an anonymous reviewer for helpful comments and suggestions on an earlier version of the manuscript. This work was partially supported by the NSF of China (grant number 11001213) and the Excellent Young Teachers Program of Xidian University.

\section{References}

[1] Atlan, M. and Leblanc, B. (2006). Time-changed Bessel processes and credit risk. Preprint.

[2] Bo, L., WANG, Y. AND YANG, X. (2011). First passage times of (reflected) Ornstein-Uhlenbeck processes over random jump boundaries. J. Appl. Prob. 48, 723-732.

[3] Bo, L., WANG, Y. AND YANG, X. (2011). Some integral functionals of reflected SDEs and their applications in finance. Quant. Finance 11, 343-348.

[4] Bo, L., Zhang, L. AND WANG, Y. (2006). On the first passage times of reflected O-U processes with two-sided barriers. Queueing Systems 54, 313-316.

[5] Bouchard, B. (2008). Optimal reflection of diffusions and barrier options pricing under constraints. SIAM J. Control Optimization 47, 1785-1813.

[6] Cox, J. C. (1975). Notes on option pricing I: constant elasticity of variance diffusions. J. Portfolio Manag. 23, $15-17$.

[7] Davydov, D. And Linestsky, V. (2001). Pricing and hedging path-dependent options under the CEV process. Manag. Sci. 47, 949-965.

[8] GöIng-Jaeschke, A. And Yor, M. (2003). A survey and some generalizations of Bessel processes. Bernoulli 9, 313-349.

[9] Goldstein, R. and Keirstead, W. (1997). On the term structure of interest rates in the presence of reflecting and absorbing boundaries. Working paper.

[10] Harrison, M. (1986). Brownian Motion and Stochastic Flow Systems. John Wiley, New York.

[11] Leung, K. S. AND KwoK, Y. K. (2007). Distribution of occupation times for constant elasticity of variance diffusion and the pricing of $\alpha$-quantile options. Quant. Finance 7, 87-94.

[12] Revuz, D. ANd Yor, M. (1991). Continuous Martingales and Brownian Motion. Springer, Berlin.

[13] Trutnau, G. (2011). Pathwise uniqueness of the squared Bessel and CIR process with skew reflection on a deterministic time dependent curve. Stoch. Process. Appl. 121, 1845-1863.

[14] Ward, A. R. And GLYNn, P. W. (2003). Properties of the reflected Ornstein-Uhlenbeck process. Queueing Systems 44, 109-123. 